\title{
Influência da idade gestacional no termo sobre o peso: estudo de coorte
}

\author{
Influence of term gestational age on weight: cohort study \\ Influencia de la edad gestacional a término sobre el peso: estudio de cohorte \\ Nathallia Serodio Michelin ${ }^{1}$ io https://orid.org/0000-0002-6750-2115 \\ Anna Paula Ferrari ${ }^{1}$ io https://orcid.org/0000-0002-5370-4349

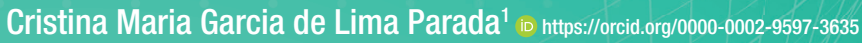

Michelin NS, Ferrari AP, Parada CM. Influência da idade gestacional no termo sobre 0 peso: estudo de coorte. Acta Paul Enferm. 2021;34:AAPE03002.

DOI

http://dx.doi.org/10.37689/actaape/2021A003002

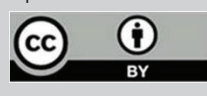

Descritores

Nascimento a termo; Peso ao nascer; Peso do feto; Peso-idade; Saúde da criança

Keywords

Term birth; Weight birth; Fetal weight; Weight by age; Child health

Descriptores

Nacimiento a término; Peso al nacer; Peso fetal; Peso por edad; Salud del niño

\section{Submetido \\ 30 de Outubro de 2019 \\ Aceito \\ 1 de Julho de 2020}

Autor correspondente

Nathallia Serodio Michelin E-mail: nathalliamichelin@gmail.com

\section{Resumo}

Objetivo: Identificar o efeito da idade gestacional no termo sobre o peso ao nascer e sua evolução no primeiro ano de vida da criança.

Método: Coorte única, concorrente, com seguimento prospectivo de um ano, realizado em Botucatu/SP. A coleta de dados foi realizada em três momentos no primeiro ano de vida da criança. Análise do peso ao nascer, aos seis e doze meses, em função da idade gestacional, incluindo potenciais variáveis de confusão foi realizada, ajustando modelos de regressão com resposta normal, após identificação das associações bivariadas com $p<0,20$. Análises executadas com 0 software SPSS v22.

Resultados: De maneira independente, crianças nascidas de termo precoce tinham, em média, $161 \mathrm{~g}$ a menos no peso ao nascer $(\beta=-161,6 ;$ IC $95 \%=-236,1--87,2 ; p<0,001)$, 350g a mais no peso aos seis meses $(\beta=0,35$; IC $95 \%=0,16-0,53 ; p<0,001)$ e $290 \mathrm{~g}$ a mais no peso aos doze meses ( $\beta=0,29 ;$ IC $95 \%=0,04-0,50 ; p=0,019$ ) que aquelas nascidas de termo completo. Achados secundários: mães com mais idade e número de consultas prénatal tiveram bebês com maior peso ao nascer; crianças com maior comprimento ao nascer tiveram maior peso aos seis e doze meses e aquelas com maior tempo de aleitamento materno tiveram menor peso aos doze meses.

Conclusão: Houve associação entre idade gestacional no termo e peso ao nascer, aos seis e doze meses. Crianças nascidas de termo precoce tiveram, de maneira independente, menor peso ao nascer e maior peso que as nascidas de termo completo aos seis e doze meses de vida.

\section{Abstract}

Objective: Identify the effect of term gestational age on birth weight and its evolution in the first year of the child's life.

Method: Single cohort, concurrent, with prospective follow-up of one year, performed in Botucatu/SP. Data were collected at three moments in the child's first year of life. To analyze the weight at birth, at six and at twelve months, in function of the gestational age, including potential confounding variables, regression models with normal response were adjusted after identifying bivariate associations with $p<0.20$. The analyses were developed using SPSS V22.

Results: Independently, early-term children were, on average, $161 \mathrm{~g}$ lighter at birth $(\beta=-161.6 ; 95 \% \mathrm{Cl}=$ $-236.1-87.2 ; p<0.001), 350 \mathrm{~g}$ heavier at six months ( $\beta=0.35 ; 95 \% \mathrm{Cl}=0.16-0.53 ; p<0.001)$ and 290 $g$ heavier at twelve months $(\beta=0.29 ; 95 \% \mathrm{Cl}=0.04-0.50 ; p=0.019)$ than full-term children. Secondary findings: mothers of older age and who attended a larger number of antenatal consultations had heavier babies at birth; longer children at birth were heavier at six and twelve months and infants breastfed longer were lighter at twelve months. 
Conclusion: Full-term gestational age was associated with birth weight, at six and twelve months. Early-term children independently showed a lower birth weight and higher weight than full-term infants at six and twelve months of age.

\section{Resumen}

Objetivo: Identificar el efecto de la edad gestacional a término sobre el peso al nacer y su evolución en el primer año de vida del niño.

Método: Cohorte única, concurrente, con seguimiento prospectivo de un año, realizado en Botucatu, estado de São Paulo. La recolección de datos se realizó en tres momentos durante el primer año de vida del niño. El peso fue analizado al nacer, a los seis y a los doce meses, en función de la edad gestacional, que incluyó potenciales variables de confusión, con el ajuste de modelos de regresión con respuesta normal, luego de la identificación de las relaciones bivariadas con $\mathrm{p}<0,20$. Los análisis se realizaron con el software SPSS v22.

Resultados: De manera independiente, los niños nacidos antes de término tenían, en promedio, $161 \mathrm{~g}$ menos de peso al nacer ( $\beta=-161,6$; IC 95 \%= -236,1$-87,2 ; p<0,001), 350 \mathrm{~g}$ más de peso a los seis meses $(\beta=0,35$; IC $95 \%=0,16-0,53 ; p<0,001)$ y $290 \mathrm{~g}$ más de peso a los doce meses $(\beta=0,29$; IC $95 \%=0,04-0,50 ; p=0,019)$ que los niños nacidos a término. Descubrimientos secundarios: madres con más edad y número de consultas de atención prenatal tuvieron bebés con más peso al nacer; niños con mayor longitud al nacer tuvieron más peso a los seis y doce meses, y los que tuvieron más tiempo de lactancia materna tuvieron un peso menor a los doce meses.

Conclusión: Se encontró relación entre edad gestacional a término y peso al nacer, a los seis y a los doce meses. Niños nacidos antes de término tuvieron, de manera independiente, menos peso al nacer y más peso que los nacidos a término a los seis y doce meses de vida.

\section{Introdução}

O peso constitui medida de viabilidade infantil, sendo importante marcador de morbimortalidade neonatal. ${ }^{(1-4)}$

Estudo de coorte realizado em FlorianópolisSC, com 15.879 nascidos vivos, encontrou associação entre peso de nascimento e óbito neonatal. (5) Além do risco de óbito precocemente, pesquisas evidenciam repercussóes do peso de nascimento ao longo da infância e vida adulta, com o aumento de síndrome metabólica em crianças e adultos associado ao peso ao nascer. ${ }^{(6-8)}$

O presente estudo tem por foco o peso infantil, considerando-se os nascimentos a termo. $\mathrm{O}$ uso da expressão nascimento a termo tende a designar um conjunto homogêneo de crianças, nascidas entre 37 e 41 semanas completas de gestação. Entretanto, cinco semanas constitui período extenso, que pode levar a diferenças relevantes nos desfechos gestacionais. Sendo assim, em 2013, o American College of Obstetricians and Gynecologists propôs nova classificação para partos não prematuros, segundo a idade gestacional no momento de sua ocorrência. Essa definição partiu de proposta conjunta com a Sociedade de Medicina Materno Fetal dos Estados Unidos da América, passando o parto a termo a ser dividido em três categorias: termo precoce ("early term"), entre 37 semanas e 38 semanas e 6 dias; termo completo ("full term"), entre 39 semanas e 40 semanas e 6 dias e termo tardio ("late term"), entre 41 semanas e 41 semanas e 6 dias, mantendo-se a classificação de pós-termo ("postterm") para crianças nascidas a partir de 42 semanas de gestaçâo. ${ }^{(9)}$

De acordo com o Comitê de Prática Obstétrica do Colégio Americano, esta mudança foi realizada para deixar claro, tanto para a comunidade médica quanto para pacientes, que não há uniformidade entre os recém-nascidos cujos partos ocorrem após 37 semanas de gestação, visto que nascer próximo de 39 semanas é importante para o completo desenvolvimento fetal antes do parto e, consequentemente, para o início de uma vida saudável. Assim, o planejamento do parto para antes de 39 semanas deve ser realizado apenas quando identificado risco significativo para a mãe ou para o feto, ou na vigência de problemas que náo possam ser controlados, como o trabalho de parto prematuro ou a rotura prematura das membranas. ${ }^{(9)}$

Considerando-se as evidências científicas sobre o pior desfecho neonatal referente ao baixo peso ao nascer e a classificação proposta para recém-nascidos de termo pelo Colégio Americano de Ginecologia e Obstetrícia, propóe-se o presente estudo, que teve por objetivo identificar o efeito da idade gestacional no termo sobre o peso ao nascer e sua evoluçáo no primeiro ano de vida da criança.

\section{Métodos}

Trata-se de coorte única e concorrente, tipo de desenho observacional no qual os participantes são classificados em expostos e não expostos, sendo 
seguidos para acompanhar a ocorrência do desfecho em determinado período de tempo, no caso, um ano. ${ }^{(10)}$ Compóe ampla pesquisa financiada pela Fundação de Amparo à Pesquisa do Estado de São Paulo (FAPESP) intitulada "Saúde da criança no primeiro ano de vida: estudo de coorte prospectiva no interior paulista”. Ambas, pesquisa mãe e recorte, foram aprovadas por Comitê de Ética em Pesquisa da Faculdade de Medicina de Botucatu/SP, número 103348/2017.

O estudo foi realizado no município de BotucatuSP, localizado na região centro-sul paulista, com população estimada de 127.328 habitantes para 2018 e parte do Departamento Regional de Saúde VI (DRS VI), Bauru, com outros 67 municípios. À época da coleta de dados, para atendimento ao parto no Sistema Único de Saúde, o município contava com um Hospital-Escola e, para assistência às gestantes conveniadas e particulares, um Hospital Privado ${ }^{(10)}$. Para atendimento ambulatorial ao recém-nascido estão disponíveis oito Unidades Básicas de modelo tradicional, doze Unidades de Saúde da Família e uma unidade neonatal, cuja finalidade é assistir a todas as crianças nascidas no município nos primeiros dias de vida, antes da vinculação destas às unidades de atenção primária de referência. ${ }^{(11)}$

A população presumida do estudo foi constituída por duplas mães-bebês residentes em Botucatu. Supondo amostragem aleatória simples, erros tipo I e II iguais a 0,05 e 0,20, respectivamente, ausência de confundidores e estimando-se $20 \%$ de perdas, a amostra final proposta foi de 520 mulheres.

Os critérios de inclusão foram nascer de termo e residir em Botucatu. Considerando-se o período estabelecido para inclusão na coorte: entre julho de 2015 e fevereiro de 2016, foram captadas, no total, 650 mães e 656 crianças. Excluídos os casos de prematuridade, natimortalidade e os gemelares, resultou em amostra inicial de 608 binômios. Porém, ao término do primeiro ano de vida, concluíram o seguimento 541 crianças (9,9\% de perdas).

Para inclusão na coorte, as mães foram abordadas e convidadas a participar na sala de espera, no momento de sua chegada para atendimento na unidade neonatal de atenção primária onde ocorreu o recrutamento. Após os esclarecimentos so- bre objetivos e procedimentos da presente pesquisa, em caso de concordância, assinaram Termo de Consentimento Livre e Esclarecido

A coleta de dados do estudo-mãe foi realizada por equipe capacitada e remunerada em três momentos durante o primeiro ano de vida da criança: presencialmente, no primeiro, sexto e décimo segundo mês.

A entrevista de inclusão na coorte foi realizada em sala privativa, em condição de conforto para a entrevistada e a criança. Foram coletados dados de identificação da mãe e recém-nascido, sociodemográficos, referentes à história gestacional pregressa, à história gestacional atual, ao parto e ao nascimento. As entrevistas de seis e doze meses ocorreram no domicílio ou na Unidade Básica de Saúde de referência, de acordo com a preferência da mãe e, entre outros, foram obtidos dados antropométricos da criança.

Os instrumentos para coleta de dados foram construídos especificamente para este estudo. Foram pré-testados, até o alcance de versão que foi considerada satisfatória pelos pesquisadores envolvidos.

A variável independente, idade gestacional, foi tratada de forma categórica politômica (37-38 semanas, 39-40 semanas e 41 semanas). Para cálculo da idade gestacional foi utilizado ultrassom precoce, realizado no primeiro trimestre de gestação ou, na ausência deste, calculou-se a idade gestacional a partir da data da última menstruação, desde que a mãe referisse ter certeza quanto a tal data.

Os desfechos em estudo foram peso ao nascer (g), e peso aos seis e doze meses de vida $(\mathrm{Kg})$.

Potenciais confundidores incluíram variáveis sociodemográficas e obstétricas maternas: idade (anos); escolaridade (anos de aprovação escolar); situação conjugal (com companheiro/sem companheiro); cor da pele autorreferida (branca/não branca); renda per capita familiar ( $\leq$ um salário mínimo/ > um salário mínimo); número de gestaçóes; número de consultas pré-natais e tipo de parto (vaginal/cesárea). Para cálculo da renda per capita familiar, inicialmente, somou-se a renda total da família em reais e dividiu-se o valor obtido pelo total de dependentes desta renda para, em seguida, considerando-se o valor do salário mínimo à época $(\mathrm{R} \$ 788,00)$, estimar-se a renda per capita familiar em salários mínimos. 
Dados do recém-nascido foram: comprimento (centímetros ao nascer, aos seis e doze meses de vida); perímetro cefálico (centímetros ao nascer, aos seis e doze meses de vida); baixo peso $(<2500 \mathrm{~g})$ ao nascer ( $\operatorname{sim} /$ não); recém-nascido pequeno para a idade gestacional ao nascer ${ }^{(12)}$ (sim/não); aleitamento materno exclusivo aos seis meses (sim, não) e duração do aleitamento materno (dias).

A análise do peso ao nascer, aos seis e doze meses de vida, em função da idade gestacional, incluindo potenciais variáveis de confundimento, foi realizada ajustando modelos de regressão com resposta normal, após identificação das associaçóes bivariadas estatisticamente mais fortes $(\mathrm{p}<0,20)$. Análises foram feitas com o software SPSS v22.

Tabela 1. Características maternas e dos recém-nascidos participantes do estudo, considerando os grupos de termo precoce $(n=250)$, completo $(n=281)$ e tardio $(n=77)$

\begin{tabular}{|c|c|c|c|}
\hline Características & $\begin{array}{c}\text { Precoce } \\
\text { Med } \\
(\text { min-max })^{\star *}\end{array}$ & $\begin{array}{c}\text { Termo } \\
\text { Completo } \\
\text { Med } \\
\text { (min-max) }^{\star \star}\end{array}$ & $\begin{array}{c}\text { Tardio } \\
\text { Med } \\
(\text { min-max })^{\star \star}\end{array}$ \\
\hline Idade materna (anos) & $28(15-42)$ & $27(14-42)$ & 25 (16-39) \\
\hline Aprovação escolar (anos) & $11(1-18)$ & $11(1-23)$ & $11(2-21)$ \\
\hline Número de gestações & $1(0-6)$ & $1(0-7)$ & $1(0-5)$ \\
\hline Número de consultas pré-natais & $8(1-15)$ & $9(1-17)$ & $11(1-15)$ \\
\hline Peso ao nascer (gramas) & $\begin{array}{c}3095 \\
(1990-4295)\end{array}$ & $\begin{array}{c}3280 \\
(2020-4700)\end{array}$ & $\begin{array}{c}3480 \\
(2285-4725)\end{array}$ \\
\hline Comprimento ao nascer (cm) & $48(42,5-52)$ & $49(40,5-57)$ & $50,5(45-54,5)$ \\
\hline \multirow[t]{2}{*}{ Perímetro cefálico ao nascer $(\mathrm{cm})$} & $34(30-40)$ & $34,5(31-42)$ & $35(31-41,5)$ \\
\hline & $\mathrm{n}(\%)$ & $\mathrm{n}(\%)$ & $\mathrm{n}(\%)$ \\
\hline Renda per capita $\leq 1 \mathrm{SM}^{*}$ & $12(4,8)$ & $21(7,5)$ & $6(7,8)$ \\
\hline Vive com companheiro & $219(87,6)$ & $246(87,5)$ & $67(87,0)$ \\
\hline Cor da pele branca & $164(65,6)$ & $169(60,1)$ & $44(57,1)$ \\
\hline Peso ao nascer $<2500 \mathrm{~g}$ & $11(4,4)$ & $4(1,4)$ & $1(1,3)$ \\
\hline Pequeno/idade gestacional & $10(4,0)$ & $21(7,4)$ & $6(7,8)$ \\
\hline Parto Cesárea & $159(64,1)$ & $119(42,3)$ & $34(44,2)$ \\
\hline Aleitamento exclusivo aos 6 meses & $7(2,8)$ & $7(2,5)$ & $2(2,6)$ \\
\hline
\end{tabular}

*SM - Salário mínimo; *^ Med (min-max) - Mediana (valor mínimo-valor máximo)

\section{Resultados}

Neste estudo foram incluídas 608 mulheres que tiveram seus filhos com idade gestacional entre $37 \mathrm{e}$ 41 semanas completas. Suas características pessoais e dos recém-nascidos constam da tabela 1 .

A maioria das mulheres era jovem, com idade mediana entre 25-28 anos; escolaridade elevada, sendo 11 anos o tempo mediano de aprovação escolar; vivia com o companheiro $(87,0 \%-87,6 \%)$ e eram de cor branca $(57,1 \%-65,6 \%)$. O nascimento por operação cesariana foi mais frequente no grupo de termo precoce $(64,1 \%)$, o peso mediano dos recém-nascidos variou entre $3.095 \mathrm{~g}$ e $3.480 \mathrm{~g}$, o baixo peso ao nascer foi mais frequente entre os nascidos de termo precoce $(4,4 \%)$ e recém-nascidos pequenos para idade gestacional estavam mais frequentemente no grupo de termo tardio (7,8\%) (Tabela 1 ).

A tabela 2 é relativa às análises brutas e ajustadas entre variáveis de interesse e o peso ao nascer.

As variáveis idade materna, vive sem companheiro, número de consultas pré-natais, termo precoce e termo tardio, apresentaram na análise bruta $\mathrm{p}<0,20$ e foram incluídas na análise ajustada. De maneira independente, crianças nascidas de termo precoce tinham, em média, $161 \mathrm{~g}$ a menos no peso ao nascer do que as nascidas de termo completo $(\beta=-161,6$; IC 95\%=-236,1- -87,2; $p<0,001)$ e as nascidas de termo tardio tinham, em média, 148 $\mathrm{g}$ a mais no peso ao nascer do que as nascidas de termo completo $(\beta=148,7$; IC 95\%= 44,2-253,0; $\mathrm{p}=0,005)$. Também de maneira independente, a cada ano de vida da mãe, o recém-nascido teve, em média, mais $6 \mathrm{~g}$ ao nascer $(\beta=5,84$; IC $95 \%=0,62$ -

Tabela 2. Análise bruta e ajustada com variáveis de interesse e peso ao nascer

\begin{tabular}{|c|c|c|c|c|c|c|}
\hline \multirow{2}{*}{ Variáveis } & \multicolumn{3}{|c|}{ Análise bruta } & \multicolumn{3}{|c|}{ Análise ajustada* } \\
\hline & $\beta$ & IC $95 \%$ & $p$-value & $\beta$ & IC95\% & $p$-value \\
\hline Idade maternal (anos) & 6,1 & $0,7-11,5$ & 0,028 & 5,8 & $0,62-11,0$ & 0,029 \\
\hline Aprovação escolar (anos) & 5,9 & $-7,5-19,3$ & 0,390 & & & \\
\hline Vive sem companheiro & $-88,8$ & $-198,4-20,9$ & 0,113 & $-40,9$ & $-145,6-63,7$ & 0,443 \\
\hline Cor da pele não branca & 25,1 & $-48,7-98,9$ & 0,505 & & & \\
\hline Renda per capita familiar & 0,09 & $-47,9-48,1$ & 0,997 & & & \\
\hline $\mathrm{N}^{0}$ de gestações & $-17,8$ & $-254,2-218,6$ & 0,883 & & & \\
\hline $\mathrm{N}^{0}$ de consultas pré-natais & 46,3 & $32,2-60,4$ & $<0,001$ & 30,0 & $15,4-44,6$ & $<0,001$ \\
\hline Termo completo & 0 & & & & & \\
\hline Termo precoce & $-191,5$ & $-256,6--117,4$ & $<0,001$ & $-161,6$ & $-236,1--87,2$ & $<0,001$ \\
\hline Termo tardio & 176,4 & $70,9-218,8$ & $<0,001$ & 148,7 & $44,2-253,0$ & 0,005 \\
\hline
\end{tabular}

*Modelo de regressão com resposta normal 
Tabela 3. Análise bruta e ajustada com variáveis de interesse e peso do bebê aos seis meses de vida

\begin{tabular}{|c|c|c|c|c|c|c|}
\hline \multirow{2}{*}{ Variáveis } & \multicolumn{3}{|c|}{ Análise bruta } & \multicolumn{3}{|c|}{ Análise ajustada* } \\
\hline & $\beta$ & IC 95\% & $p$-value & $\beta$ & IC95\% & $p$-value \\
\hline Idade maternal (anos) & 0,0 & $-0,0-0,1$ & 0,836 & & & \\
\hline Aprovação escolar (anos) & 0,0 & $-0,0-0,1$ & 0,267 & & & \\
\hline Vive sem companheiro & 0,1 & $-0,1-0,4$ & 0,324 & & & \\
\hline Cor da pele não branca & 0,0 & $-0,2-0,2$ & 0,978 & & & \\
\hline Renda per capita familiar & 0,02 & $-0,09-0,14$ & 0,643 & & & \\
\hline Recém-nascido pequeno para a idade gestacional & $-0,79$ & $-1,16--0,42$ & $<0,001$ & $-0,20$ & $-4,7-0,04$ & 0,054 \\
\hline Comprimento ao nascer & 0,1 & $0,1-0,2$ & $<0,001$ & 0,20 & $0,16-0,53$ & $<0,001$ \\
\hline Duração do aleitamento materno & 0,0 & $-0,001-0,002$ & 0,680 & & & \\
\hline Termo completo & 0 & & & & & \\
\hline Termo precoce & 0,1 & $-0,1-0,3$ & 0,165 & 0,35 & $0,16-0,53$ & $<0,001$ \\
\hline Termo tardio & 0,3 & $0,1-0,6$ & 0,021 & 0,13 & $-0,12-0,40$ & 0,310 \\
\hline
\end{tabular}

${ }^{*}$ Modelo de regressão com resposta normal

Tabela 4. Análise bruta e ajustada das variáveis de interesse e peso da criança aos doze meses

\begin{tabular}{|c|c|c|c|c|c|c|}
\hline \multirow{2}{*}{ Variáveis } & \multicolumn{3}{|c|}{ Análise bruta } & \multicolumn{3}{|c|}{ Análise ajustada* } \\
\hline & $\beta$ & IC $95 \%$ & $p$-value & $\beta$ & IC95\% & $p$-value \\
\hline Idade maternal (anos) & 0,0 & $-0,1-0,0$ & 0,726 & & & \\
\hline Aprovação escolar (anos) & 0,0 & $-0,1-0,1$ & 0,106 & 0,028 & $-0,01-0,06$ & 0,156 \\
\hline Vive sem companheiro & 0,0 & $-0,3-0,4$ & 0,896 & & & \\
\hline Cor da pele não branca & $-0,1$ & $-0,3-0,2$ & 0,685 & & & \\
\hline Renda per capita familiar & 0,06 & $-0,08-0,21$ & 0,372 & & & \\
\hline Recém-nascido pequeno para a idade gestacional & $-0,85$ & $-1,3--0,40$ & $<0,001$ & $-0,16$ & $-0,61-0,27$ & 0,497 \\
\hline Comprimento ao nascer & 0,2 & $0,1-0,3$ & $<0,001$ & 0,24 & $0,18-0,30$ & $<0,001$ \\
\hline Duração do aleitamento materno & $-0,002$ & $-0,003-0,0003$ & 0,114 & $-0,002$ & $-0,004--0,001$ & $<0,001$ \\
\hline Termo completo & 0 & & & & & \\
\hline Termo precoce & 0,1 & $-0,2-0,3$ & 0,451 & 0,29 & $0,04-0,50$ & $<0,019$ \\
\hline Termo tardio & 0,2 & $-0,2-0,5$ & 0,320 & $-0,06$ & $-0,37-0,25$ & 0,707 \\
\hline
\end{tabular}

*Modelo de regressão com resposta normal

$11,0 ; \mathrm{p}=0,029)$ e a cada consulta pré-natal a mais os recém-nascidos apresentaram mais $30 \mathrm{~g}$ ao nascer: $\beta=30,0 ;$ IC 95\%= 15,4-44,6; $p<0,001$ (Tabela 2). A tabela 3 refere-se ao peso do bebê aos seis meses de vida.

As variáveis recém-nascido pequeno para a idade gestacional, comprimento ao nascer, termo precoce e termo tardio apresentaram na análise bruta $\mathrm{p}<0,20$ e foram incluídas na análise ajustada. De maneira independente, crianças nascidas de termo precoce tinham, em média, $350 \mathrm{~g}$ a mais no peso aos seis meses que aquelas nascidas de termo completo $(\beta=0,35$; IC 95\%=0,16-0,53; $\mathrm{p}<0,001)$. Também de maneira independente, houve associação entre comprimento ao nascer e peso aos seis meses de vida, sendo que a cada centímetro a mais ao nascer, as crianças tinham, em média, mais $200 \mathrm{~g}$ aos seis meses: $\beta=0,20$; IC $95 \%=0,16-0,53 ; p<0,001$ (Tabela 3). A tabela 4 é relativa ao peso da criança aos 12 meses de vida.

Escolaridade, recém-nascido pequeno para a idade gestacional e comprimento ao nascer apresen- taram $\mathrm{p}<0,20$ na análise bruta e foram incluídas na análise ajustada, assim como as variáveis de interesse: termo precoce e termo tardio. De maneira independente, crianças nascidas de termo precoce tinham, em média, $290 \mathrm{~g}$ a mais no peso aos 12 meses do que aquelas nascidas de termo completo $(\beta=0,29 ;$ IC 95\%=0,04-0,50; $p=0,019)$. Também de maneira independente, houve associação entre comprimento ao nascer e duração do aleitamento materno e peso aos 12 meses, sendo que a cada centímetro a mais ao nascer, as crianças tinham, em média, mais $240 \mathrm{~g}$ aos 12 meses: $\beta=0,24$; IC 95\%= $0,18-0,30 ; \mathrm{p}<0,001$ e a cada dia a mais na duração do aleitamento materno as crianças tinham, em média, menos 2g: $\beta=-0,002$; IC 95\%= -0,004- 0,001 ; $\mathrm{p}<0,001$ (Tabela 4).

\section{Discussão}

De maneira independente, ao nascimento, crianças nascidas de termo precoce tiveram, em média, 
menos $161 \mathrm{~g}$ que as nascidas de termo completo, enquanto que as nascidas de termo tardio tiveram mais $148 \mathrm{~g}$ que as nascidas de termo completo. Também de maneira independente, crianças nascidas de termo precoce tiveram, em média, $350 \mathrm{~g}$ a mais no peso aos seis meses e $290 \mathrm{~g}$ a mais no peso aos doze meses de vida, quando comparadas àquelas nascidas de termo completo.

Sobre o peso ao nascer, os resultados obtidos são concordantes com a literatura científica, havendo evidências da relevância de cada semana a mais no tempo de gestação para incremento do peso do recém-nascido, ${ }^{(12,13)}$ o que explica, inclusive, o maior peso entre os nascidos de termo tardio, quando comparados aos de termo completo. Estudo de base populacional que avaliou o crescimento fetal e o tamanho de recém-nascidos em oito países, demonstrou que as curvas para o peso ao nascer sáo diretamente proporcionais à idade gestacional. ${ }^{(12)}$ No Brasil, estudo realizado com dados do Sistema de Informaçóes de Nascidos Vivos (SINASC), entre os anos de 2001 a 2010, evidenciou que a proporção de recém-nascidos com baixo peso diminuiu à medida que a duração da gestação aumentou. ${ }^{(13)}$

A taxa de baixo peso no presente estudo foi, em geral, baixa, situação esperada por tratar-se de crianças nascidas de termo. Porém, entre os nascidos de termo precoce, esta foi três vezes maior que a observada entre os nascidos de termo completo ou tardio, indicando a importância das gestaçóes de termo evoluírem para parto apenas após 39 semanas.

Sabe-se que o baixo peso ao nascer constitui condição de risco para crianças em geral. Entretanto, é relevante considerar o peso em relação à idade gestacional, visto que na vigência de adequação o prognóstico é melhor, mesmo em condição de baixo peso. A explicação está no crescimento pós-natal compensatório, em resposta à desaceleração no ritmo ponderal de crescimento da criança durante a gestação, pois a medida em que se corrige a causa do problema e em condiçôes ambientais adequadas, o organismo passa a crescer em velocidade superior ao esperado para a idade, de forma que essas crianças chegam ao peso normal para a idade ainda no primeiro ano de vida. ${ }^{(14)}$ Estudo com recém-nascidos pré-termo, que investigou os padróes de ama- mentação e a evolução do ganho de peso ponderal, demonstrou que quanto menor a idade gestacional e/ou o peso de nascimento, maior o ganho ponderal pós natal. ${ }^{(15)}$

Diferentemente do peso ao nascer, encontrou-se para o peso aos seis e doze meses, de maneira independente, maiores valores entre os nascidos de termo precoce do que entre os nascidos de termo completo ou tardio, indicando aceleração no ganho de peso entre aqueles que nascem de termo, mas com menor idade gestacional e peso. Este achado nos remete à discussão já mencionada sobre a aceleração no ganho de peso após o nascimento em condiçôes de prematuridade e/ou de crianças pequenas para a idade gestacional. ${ }^{(14)}$ Entretanto, a situação encontrada entre os bebês de termo precoce é inversa, na medida em que têm entre 37-38 semanas de gestação e porque neste grupo há, proporcionalmente, a metade dos casos de crianças classificadas como pequenas para a idade gestacional, quando comparadas aos grupos de termo tardio e completo.

Considera-se que, embora a explicação sobre a aceleração compensatória do ganho de peso, até o momento, voltar-se especificamente às crianças prematuras e de baixo peso para a idade gestacional, pode se aplicar aos nascidos de termo precoce, inclusive porque alguns pesquisadores apontam esse grupo com respostas mais próximas às dos prematuros tardios do que dos nascidos de termo completo. Nesse contexto, estudo transversal realizado na França no ano de 2010, investigou os fatores de risco para crianças nascidas prematuras tardias e de termo precoce, tomando por referência nascidos de termo completo e concluiu que os dois grupos apresentavam os mesmos fatores de risco associados devendo, portanto, ter estratégias comuns de prevenção. ${ }^{(16)}$

Assim, outros estudos devem ser conduzidos, com amostras maiores, de forma a confirmar se o aumento do peso de recém-nascidos de termo precoce é, realmente, mais acelerado que o observado entre os demais nascidos de termo, condição relevante pelas evidências na literatura científica de associação entre baixo peso ao nascer e desenvolvimento de Síndrome Metabólica em adultos. ${ }^{(6-8,17,18)}$

São achados secundários deste estudo: de maneira independente, houve associação diretamen- 
te proporcional entre idade materna e número de consultas pré-natais e o peso ao nascer. Também foi diretamente proporcional a associaçáo entre comprimento ao nascer e peso aos seis e doze meses, mas foi inversa a associação entre duração do aleitamento materno e peso aos doze meses.

Com relação ao peso ao nascer, os resultados obtidos relativos à idade materna estáo de acordo com a literatura científica, pois estudos com essa temática relacionam o aumento do peso das crianças ao aumento da idade materna, com relação inversa apenas nos extremos de idade. ${ }^{(19-21)}$ Sobre o número de consultas pré-natais, pode estar relacionado à maior duração da gestação, visto que quanto mais prolongada, mais consultas são agendadas, sendo que a partir de 36 semanas de gestação estão indicados, pelo menos, retornos semanais. ${ }^{(22)}$

Sobre o peso aos seis e doze meses, a associação positiva com a estatura ao nascer também foi encontrada em dois estudos realizados no estado de Minas Gerais, em que as crianças que apresentavam maior comprimento ao nascer apresentaram maior velocidade de ganho de peso nos primeiros seis ${ }^{(23)}$ ou doze meses de vida. ${ }^{(24)}$ Sobre o fato de crianças com maior duração do aleitamento materno apresentarem menor peso aos doze meses, pode decorrer do uso mais prolongado de fórmulas lácteas infantis, já que essas podem apresentar valores de lipídios e carboidratos excessivo. ${ }^{(25)}$

Constitui limitação do presente estudo, o fato de a captação dos binômios para participação da coorte ter se dado em unidade básica de triagem neonatal, que atende crianças no primeiro mês de vida, assim, podem ter sido pouco captadas mães e crianças que apresentaram intercorrências que poderiam impactar nos desfechos estudados.

\section{Conclusão}

Este estudo evidenciou a influência da idade gestacional das crianças nascidas de termo precoce sobre o peso ao nascer, aos seis e dose meses: o peso foi $161 \mathrm{~g}$ menor ao nascer, $350 \mathrm{~g}$ maior aos seis meses e $290 \mathrm{~g}$ maior aos doze meses de vida, quando comparadas às crianças nascidas de termo completo.
No entanto, estudos com esse enfoque são escassos, de forma que para promoção de ampla discussão sobre essa temática, outras pesquisas deverão ser conduzidas.

\section{Agradecimentos}

Apoio financeiro da Fundação de Amparo à Pesquisa do Estado de São Paulo (FAPESP), Brasil, processo ${ }^{\circ}$ 2015/03256-1 e Coordenação de Aperfeiçoamento de Pessoal de Nível Superior (CAPES).

\section{Referências}

1. Veloso FC, Kassar LM, Oliveira MJ, Lima TH, Bueno NB, Gurgel RQ, et al. Analysis of neonatal mortality risk factors in Brazil: a systematic review and meta-analysis of observational studies. J Pediatr (Rio J). 2019;95(5):519-30.

2. Madden JV, Flatley CJ, Kumar S. Term small-for-gestational-age infants from low-risk women are at significantly greater risk of adverse neonatal outcomes. Am J Obstet Gynecol. 2018;218(5):525.e1-9.

3. Tabet M, Flick LH, Xian H, Chang JJ. The difference in sibling birthweight and neonatal death: a population-based cohort study. Am J Perinatol. 2019;36(5):498-504.

4. Gaiva MA, Fujimori E, Sato APS. maternal and child risk factors associated with neonatal mortality. Texto Contexto Enferm 2016;25(4):1-9.

5. Garcia LP, Fernandes CM, Traebert J. Risk factors for neonatal death in the capital city with the lowest infant mortality rate in Brazil. J Pediatr (Rio J). 2019;95(2):194-200.

6. Heidemann LA, Procianoy RS, Silveira RC. Prevalence of metabolic syndrome-like in the follow-up of very low birth weight preterm infants and associated factors. J Pediatr (Rio J). 2019;95(3):291-7.

7. Ribeiro AM, Lima MC, de Lira Pl, da Silva GA. [Low birth weight and obesity: causal or casual association?]. Rev Paul Pediatr. 2015;33(3):341-9. Portuguese.

8. Huang YT, Lin HY, Wang CH, Su BH, Lin CC. Association of preterm birth and small for gestational age with metabolic outcomes in children and adolescents: A population-based cohort study from Taiwan. Pediatr Neonatol. 2018;59(2):147-53.

9. The American College of Obstetricians and Gynecologists. ACOG Committee Opinion No 579: definition of term pregnancy. Obstet Gynecol. 2013;122(5):1139-40.

10. Oliveira RL, Fonseca CR, Carvalhaes MA, Parada CM. Avaliação da atenção pré-natal na perspectiva dos diferentes modelos na atenção primária. Rev Lat Am Enferm. 2013;21(2):1-8.

11. Brasil. Secretaria de Atenção à Saúde. Ficha Estabelecimento- Tipo de Estabelecimento Centro de Saúde/Unidade Básica [Internet]. Brasília (DF): Cadastro Nacional de Estabelecimentos de Saúde; 2019. [citado 2020 Jun 30]. Disponível em: http://cnes2.datasus.gov.br/Mod_Ind_ Unidade. asp?VEstado $=35 \&$ VMun $=350750$ 
12. Villar J, Cheikh Ismail L, Victora CG, Ohuma EO, Bertino E, Altman DG, et al.; International Fetal and Newborn Growth Consortium for the 21st Century (INTERGROWTH-21st). International standards for newborn weight, length, and head circumference by gestational age and sex: the Newborn Cross-Sectional Study of the INTERGROWTH-21st Project. Lancet. 2014;384(9946):857-68.

13. São Paulo (cidade). Secretaria Municipal da Saúde. Coordenação de Epidemiologia e Informação. 0 perfil dos nascimentos na cidade de São Paulo. Dez anos do SINASC. Boletim CEINfo. 2012;4(4):1-39.

14. Kopec G, Shekhawat PS, Mhanna MJ. Prevalence of diabetes and obesity in association with prematurity and growth restriction. Diabetes Metab Syndr Obes. 2017;10:285-95.

15. Sassá AH, Schmidt KT, Rodrigues BC, Ichisato SM, Higarashi IH, Marcon SS. Bebês pré-termo: aleitamento materno e evolução ponderal. Rev Bras Enferm. 2014;67(4):594-600.

16. Delnord M, Blondel B, Prunet C, Zeitlin J. Are risk factors for preterm and early-term live singleton birth the same? A population-based study in France. BMJ Open. 2018;8(1):e018745.

17. Sipola-Leppänen $M$, Vääräsmäki $M$, Tikanmäki $M$, Matinolli $H M$, Miettola S, Hovi P, et al. Cardiometabolic risk factors in young adults who were born preterm. Am J Epidemiol. 2015;181(11):861-73.

18. Zachariassen LH. Corrigendum to Toftlund et al. Early Nutrition and Signs of Metabolic Syndrome at 6 years of age in Children Born Very Preterm. Am J Clin Nutr 2018;107:717-724. Am J Clin Nutr. 2019;110(2):530-1.
19. Nunes FB, Silva PC, Barbosa TL, Lopes ML, Farias RA, Silva EL. Influence of maternal age in perinatal conditions in live births of São Luís, Maranhão. Rev Pesq Cuid Fundam Online. 2020;(12):292-9.

20. Moreira ME, Silva CL, Freitas RF, Macêdo MS, Lessa AD. Socioeconomic and gestational determinants of birth weight of term infants. Medicina (Ribeirao Preto Online). 2017;50(2):83-90.

21. Dowell CM, Mejia GC, Preen DB, Segal L. Low birth weight and maternal incarceration in pregnancy: A longitudinal linked data study of Western Australian infants. SSM Popul Health. 2019;7(Apr):100324.

22. Brasil. Ministério da Saúde. Atenção à saúde do recém-nascido [Internet]. Brasília (DF):Ministério da Saúde; 2014. Vol.1. 192 p. [citado 2020 Jun 30]. Disponível em: http://www.redeblh.fiocruz.br/media/ arn_v1.pdf

23. Vieira $S A$, Magalhães $T C$, Ribeiro $A Q$, Priore $S E$, Franceschini SC, Sant'Ana LF. Franceschini S do CC, Sant'Ana LF da R. Fatores associados às velocidades de ganho de peso e de comprimento nos primeiros seis meses de vida. Cad Saude Colet. 2015;23(3):309-15.

24. Camargos AC, Azevedo BN, da Silva D, Mendonça VA, Lacerda AC. Prevalência de sobrepeso e de obesidade no primeiro ano de vida nas Estratégias Saúde da Família. Cad Saude Colet. 2019;27(1):32-8.

25. Freitas JA, Junqueira GP, Alves MS, Mansur LR. Análise e comparação da composição nutricional de fórmulas infantis comercializas no município de Campos dos Goytacazes - RJ. Rev Conhecendo Online Ciênc Saúde Biol. 2019;5(1):31-47. 